\title{
Validation test on 3D heart phantom for mitral valve leaflet tracking
}

\author{
Lina Farhana Mahadi ${ }^{1}$, Nabilah Ibrahim ${ }^{2}$, Shahnoor Shanta ${ }^{3}$, Hideyuki Hasegawa ${ }^{4}$ \\ ${ }^{1,2}$ Faculty of Electrical and Electronic Engineering, Universiti Tun Hussein Onn Malaysia, Malaysia \\ ${ }^{3}$ Institute of Technology Sligo, Ash Ln, Bellanode, Sligo, Ireland \\ ${ }^{4}$ Faculty of Engineering, University of Toyama, Gofuku, Toyama, Japan
}

\begin{tabular}{l} 
Article Info \\
\hline Article history: \\
Received Jan 21, 202 \\
Revised Mar 20, 202 \\
Accepted Mar 29, \\
\hline Keywords: \\
Echocardiography \\
Heart phantom \\
KLT algorithm \\
Mitral valve
\end{tabular}

\begin{abstract}
Mitral valve movement is essential to be identified in order to monitor the abnormality of blood flow in right side of heart. The tracking of mitral valve has seldom been investigated since it required high temporal rate to scan the echocardiography images and it depends on the operator to capture the lowspeckle and noise images. This study presents the validation experiment performed on heart phantom made of thermoplastic polyurethane (TPU) filament which the objective is to validate the previous features tracking technique implemented in mitral valve locating in video frames using kanadelucas-tomasi (KLT) algorithm. The outcome was able to automatically detect the edge of mitral valve and thus in future, it manages to predict the flowing of blood pattern. An in-vitro experiment was conducted which involved a valve phantom scanning in water tank that connected to water pump. It was found in this study that the technique capable to detect and visualize the mitral valve up to 59 frames in 2.36 seconds by tracking the features of minimum eigenvalue within the selected region. It was also produced a good agreement of valve distance between the true value and the measured one, which achieved the minimum of $88 \%$ similarity. This yielded the validation of the proposed technique to track and visualize the mitral valves.
\end{abstract}

This is an open access article under the CC BY-SA license.

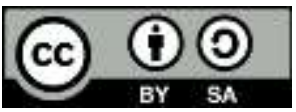

\section{Corresponding Author:}

Nabilah Ibrahim

Department of Electrical and Electronic Engineering

Universiti Tun Hussein Onn Malaysia

86400, Batu Pahat, Johor, Malaysia

Email: nabilah@uthm.edu.my

\section{INTRODUCTION}

Blood circulation starts when blood flows from the atria into the ventricle which is then the ventricle starts to expand. Right after the ventricles filled with the oxygenated or deoxygenated blood, the ventricles pump the blood into the large arteries to be transferred to the whole body and to the lung for the deoxygenated blood exchange. Number of studies reported [1], [2] the left ventricle is located at the bottom left portion of the heart below the left atrium, separated by the mitral valve. Various conditions may affect the left ventricle and interfere with its proper functioning. The most common are left ventricular hypertrophy, which causes enlargement and hardening of the muscle tissue that makes up the wall of the left ventricle, usually as a result of uncontrolled high blood pressure. In addition, the mitral valve regurgitation or incompetence is also frequently occurring which defined as improper close of valve when the heart pumps the bloods out. This leads to the blood leaking into the atrium from the ventricle due to mitral valve prolapse [3]. Mitral valve prolapse is the condition when the leaflets of the mitral valve bulge (prolapse) into the left atrium during the heart contraction [4]. Therefore, during the contraction, the valve allows blood to flow in 
two opposite directions. Supposedly, the blood will flow from the ventricle through the aortic valve, however, in this case some of the blood will flow back into the atrium. Thus, the blood volume and pressure in the area will increase due to the leakage. For advanced method, ultrasound medical imaging has been widely used as diagnostic technique. Since the ultrasound imaging technique is non-invasive, low cost and save time, some take the advantage of the doppler technique to measure the blood flow velocity in heart [5][7]. Conventional method that applied doppler technique did provide the imaging blood flow either employing continuous wave, pulse wave or color wave [8]. However, the image acquisition is mostly operator dependent, which could be argued on lack of reproducibility results. Despite the measurement of blood flow velocity, researchers found that the study of the factor that trigger the velocity in heart is more reliable, which focus on valve patency behavior [9]-[11]. The evaluation on patients with mitral valve disease is one of the most promising clinical application of echocardiography. This work proposed a technique to assess the mitral valve by tracking it using object tracking technique on ultrasound video. This technique could prevent the mistracking point during the short-time frame. Object tracking is a process of locating moving objects in the consecutive video frames. In other applications, real time object tracking is a challenging problem in the field of computer vision [12], motion-based recognition, automated surveillance [13], traffic monitoring [14], medical imaging [15] augmented reality [16], and video editing [17], [18]. To be focus, the important issues related to feature tracking are the use of appropriate image features, selection of motion models, and detection of objects. Thus, this paper implements the idea of tracking the mitral valve in the ultrasound video by employing kanade-lucas-tomasi (KLT) algorithm or so-called object tracking technique. This technique is able to track the motion based on feature point in the selected region. Moreover, the validation performance is obtained through the in-vitro experiment to support the accuracy of the implemented technique.

\section{RESEARCH METHOD}

\subsection{Feature tracking selection}

KLT technique is employed to track the mitral valve in video frames. The technique is able to track a set of feature points across the video frames [19] based on the analysis of minimum eigenvalues. In general, video frames could be defined as any function of three variables $I(x, y, t)$, where the space variables $x$ and $y$ as well as the time variable $t$ are discrete and suitably bounded. However, images taken at near time instants are usually strongly related to each other, because they refer to the same scene taken from only slightly different viewpoints [20]. This could be expressed as follows:

$$
I(x, y, t+\tau)=I(x-\xi, y-\eta, t)
$$

The next image taken at time $(t+\tau)$ can be obtained by moving every point in the current image. The value of motion $d=(\xi, \eta)$ is called the displacement of the point at $x=(x, y)$ between time instants $t$ and $(t+\tau)$.

For the region (mitral valve) tracking, $2 \times 2$ coefficient matrix $G$ of the system must be both above the image noise level and stable. In practice, if the smaller eigenvalue is sufficiently large to meet the noise level, the matrix $G$ is normally stable. This is due to the fact that the intensity variations in a window are bounded by the maximum allowable pixel value. Thus, the greater eigenvalue cannot be arbitrarily large. Moreover, if the two eigenvalues of $\mathrm{G}$ are $\lambda 1$ and $\lambda 2$, the condition of $\min (\lambda 1, \lambda 2)>\lambda$ should be satisfied, where $\lambda$ is a predefined threshold. The threshold $\lambda$ is determined by measuring the eigenvalues for the region in images of approximately uniform brightness. This technique could provide a lower bound for $\lambda$. Then, a set of various types of features, such as corners and highly textured regions is selected to obtain an upper bound for $\lambda$.

\subsection{Ultrasound image acquisition}

Ultrasound heart images were obtained by the S5-1 sector probe, connected to ultrasonic diagnosis equipment Philips iE33, which located in medical imaging laboratory pantai hospital klang. A subject who suffers from mitral valve disease was selected in this study. The images were scanned by a cardiovascular technologist of pantai hospital klang, from the left parasternal window. The parasternal long axis (PLAX) view as shown in Figure 1(a) is obtained by placing the transducer on the three to four left intercostal spaces close to the sternum with the beam oriented toward the patient's right shoulder. This orientation is slicing through the heart on a long axis from base to apex. Based on the image, it can be seen that mitral valve (MV) is the boundary between the left atrium (LA) and left ventricle (LV). The mitral valve would act as open and close due to the pressure intake from the left ventricle wall and also from the velocity of blood flowing. Image processing is conducted by using MATLAB platform. Since the ultrasound image can only be read by dicom software specialize for medical image purpose, the recorded video file is converted into avi format before extracted into frame images. Figure 1(b) shows the Dicom interface for reading the ultrasound image 
and the steps to export the file to video avi format. Here, in order to preserve the originality of the recorded raw image, the size of the video is set up similar to the original one without compression level.

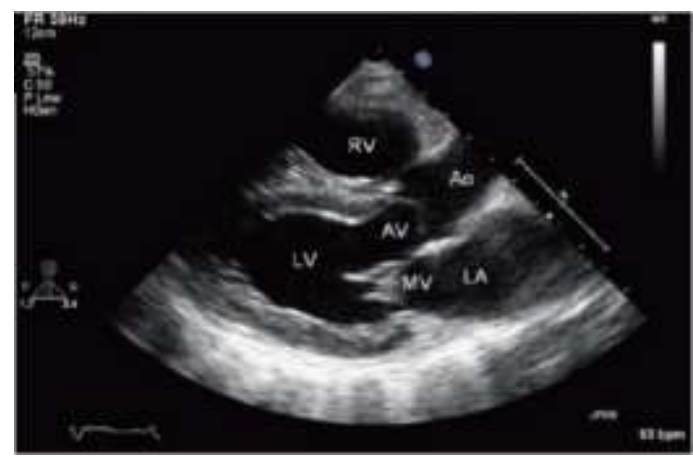

(a)

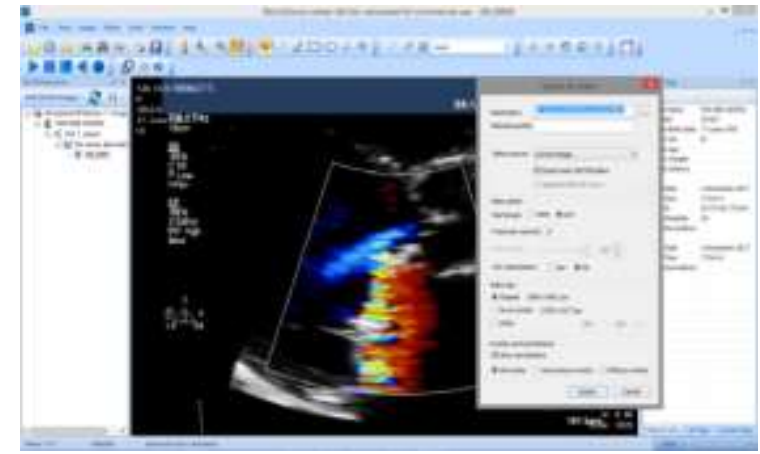

(b)

Figure 1. (a) The parasternal long axis (PLAX) view. LA, LV, and RV are stand for left atrium, and left/right ventricle, respectively, while MV, AV and Ao are mitral valve, aortic valve and aorta;

(b) Dicom interface for reading the ultrasound image and video conversion format

The region of interest which focus on mitral valve as the feature was extracted by bounding box, as shown in Figure 2. Once the region of the mitral valve was detected, the feature-points were selected based on the calculation of eigenvalue. The minimum eigenvalue was calculated within the bounded box to find the edge of the mitral valve, so that it could give the guidance for the calculation in tracking the mitral valve on the next frame by setting the standard detected mitral valve. The detected points in standard image are marked by the white ' + ' symbols as shown in Figure 3. Next, the current image is tracked from the previousframe to detect the features. The result of detected mitral valve on ultrasound heart image have been reported in [21].

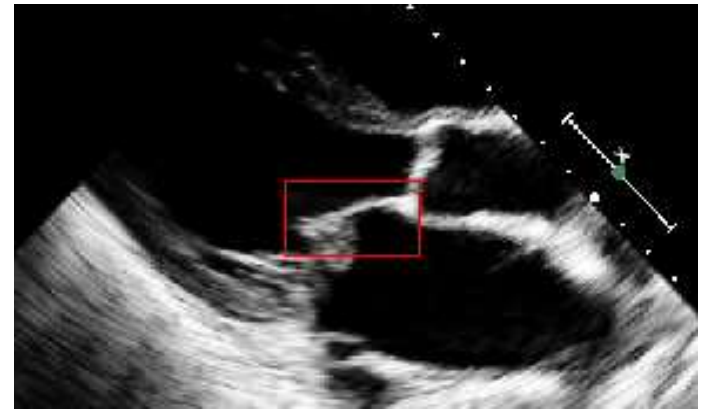

Figure 2. Detection of mitral valve region

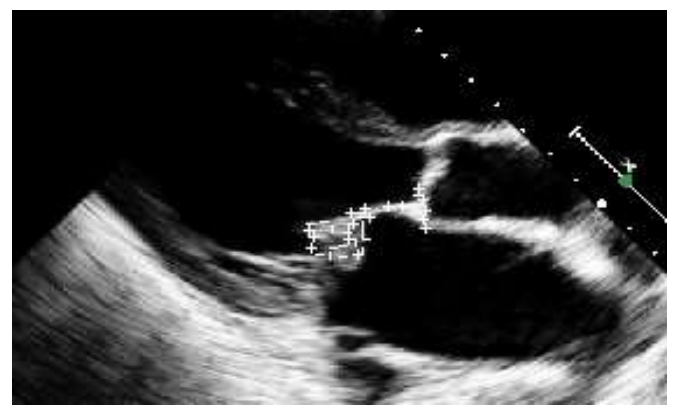

Figure 3. Detection of mitral valve edge

\subsection{Validation Test for Mitral Valve Tracking}

For validation stage, 3-dimensional (3D) heart phantom is used to mimic the heart structure and function. The heart phantom was modelled by using mathematica design tool as shown in Figure 4 based on the real human heart. The distance of valves was constructed at $25.5 \mathrm{~mm}$ measured by millimeter measurement on the phantom. The phantom was created as multi material version comprising arteries, veins, atrium, ventricle, valves and papillaries where the ventricle chamber was included with some holes which makes it possible to observe the valves and papillaries. This $3 \mathrm{D}$ heart phantom was printed by using thermoplastic polyurethane (TPU) filament which physically between plastic and rubber. This material is elastic in nature allowing the plastic to be stretched and flexed easily. However, the valves are made by using silicone which has more flexible properties than TPU. The experiment was conducted as illustrated in Figure 5. The experiment that involved the developed heart phantom was emerged into water and connected to water pump to mimicking the blood flow with water. The phantom was scanned by ultrasound transducer connected to computer and modified ultrasound machine SYS0007. 


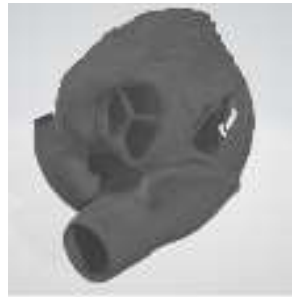

(a)

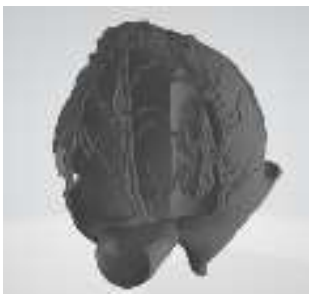

(b)

Figure 4. 3D heart in whole with; (a) front; (b) back views

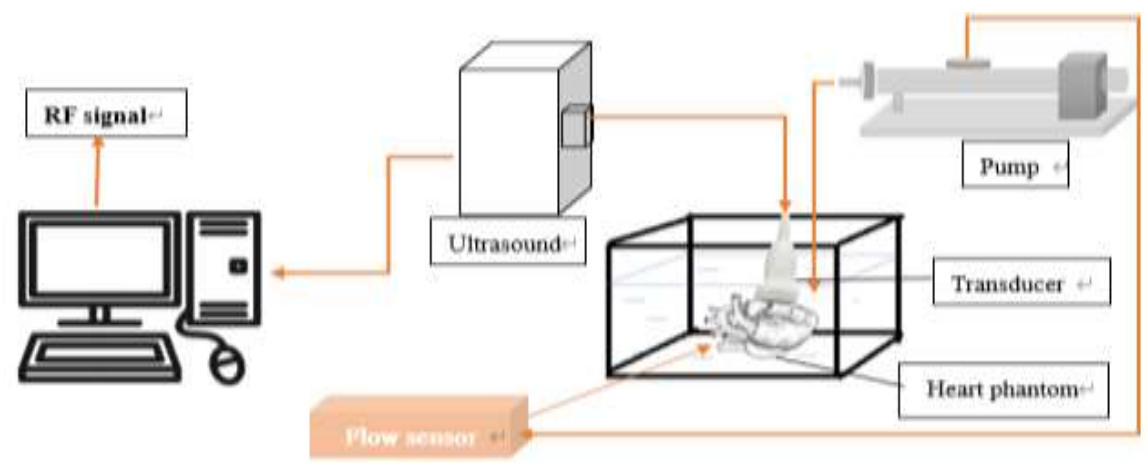

Figure 5. Illustration of experiment setup for the heart phantom scanning

\section{RESULTS AND DISCUSSION}

This section demonstrates the KLT detection performance, and at the same time, provides the distance measurement of heart valves phantom due to the movement and position changes over time. Figure 6(a) shows an ultrasound heart phantom image with red box indicates the selected region of interest. From the image that prior to the injection water pump, the true distance of valve measured by the pixel is $25.5 \mathrm{~mm}$ which $100 \%$ similar to that measured at the valve phantom itself, thus validate the ultrasound image performance. Within the selected area, the detected corner in interest region is marked by the white ' + ' symbols as shown in Figure 6(b). Then, the point tracker system will track the mitral valve frame by frame as shown in Figure 7(a)-(i). The detected point is then labelled with green '+' symbol. According to the result, it can be seen that the tracker can effectively track the mitral valve within the region of interest although the accuracy decreases over time. Noted that the valve moves due to the water mimicking the blood flow. During cardiac cycle, mitral valve tends to change its shape and positions. Therefore, the contraction of mitral valve effecting the distance of valves thus distract the tracking system. This reason caused the unrecognization of eigenvalue calculation.

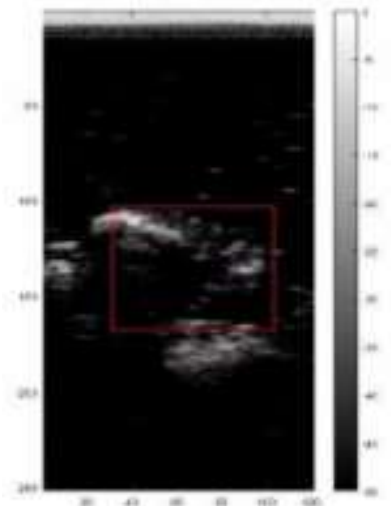

(a)

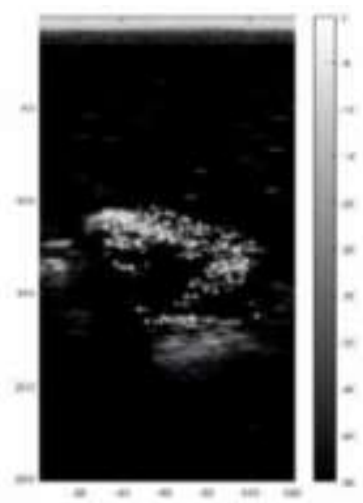

(b)

Figure 6. (a) Selected region of interest; (b) Detected corner in interest region 


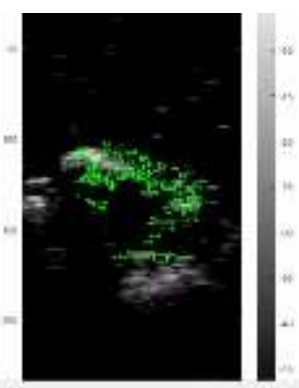

(a)

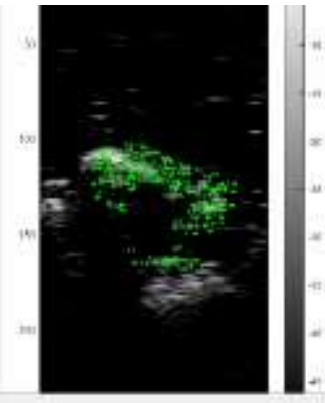

(e)

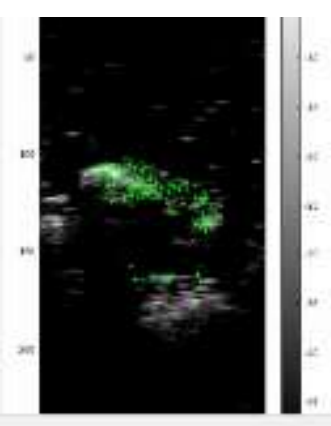

(i)

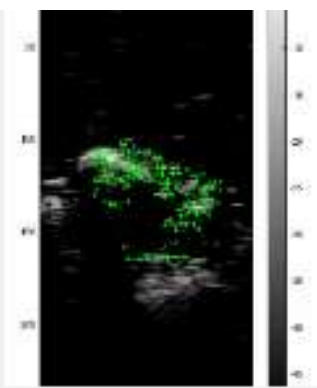

(b)

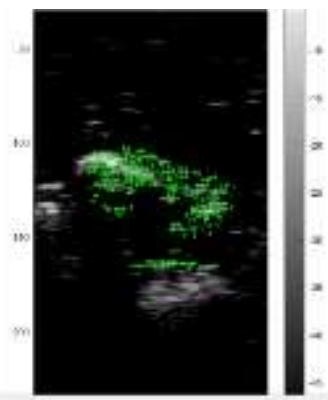

(f)

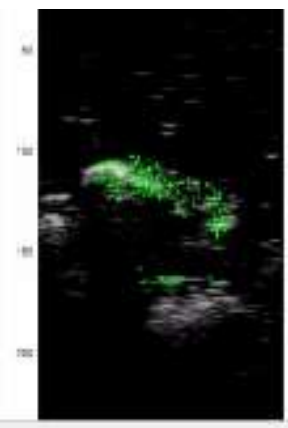

(j)

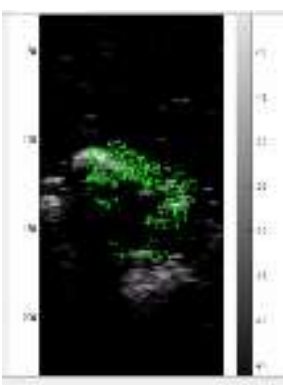

(c)

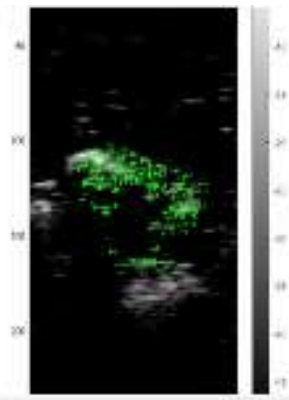

(g)

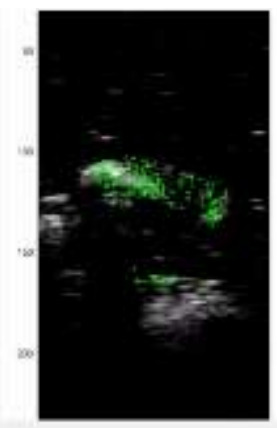

(k)

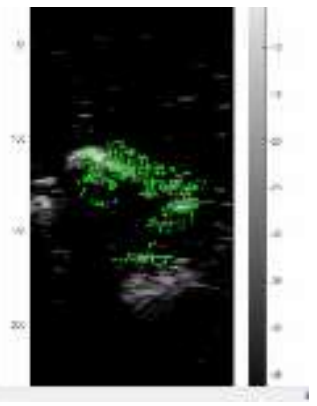

(d)

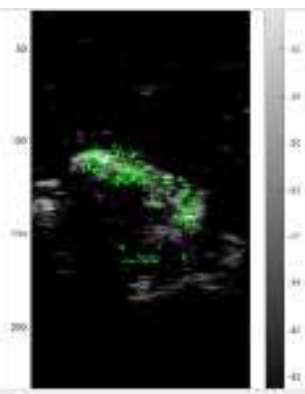

(h)

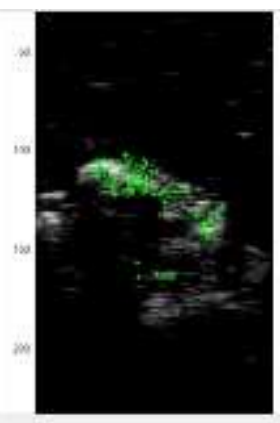

(1)

Figure 7. Point detected; (a) Frame 2; (b) Frame 5; (c) Frame 6; (d) Frame 7; (e) Frame 8; (f) Frame 9; (g) Frame 15; (h) Frame 24; (i) Frame 33; (j) Frame 42; (k) Frame 48; (l) Frame 59

In addition, the measurement of valve distance is shown in Figure 8 for selected frame images. Here, the calculation of the distance in millimeter has been discussed in [22]. The difference in distance between valves is shown by graph in Figure 9 in pixel value. The fluctuation of the graph is the resulting of contraction of the valve phantom and the water flow. In this experiment, the tracking system are able to detect the mitral valve accurately up to 59 frames or equivalent to $2.36 \mathrm{~s}$. In the beginning, the distance of the valves was measured as 96.56 pixel that was $25.55 \mathrm{~mm}$. This value is the highest distance and it results with the minimum of 85.33 pixel which was $22.58 \mathrm{~mm}$ in $27^{\text {th }}$ frame. The points that being selected to be measured are the one that visible enough so that it could be manually determined and also by referring to the pixel value.

From a physical point of view, the eigen-values or eigen-vectors represent the information space as a rotated hyper-ellipsoid. The eigen vectors capture the underlying hyper-rotation matrix while the eigen values capture the length of the axes of this hyper-ellipsoid. This hyper-ellipsoid conveys a very complex interactive relationship between the camera geometry, surface materials, brightness that led to the composition of pixel values and their contextual relationships. This concept however has been reported in [23], [24]. In this work, the eigen values are used to classify the corner of image points. Therefore, the eigenvalue measurement will provide high accuracy in consecutive image. This technique gives advantages to process consecutives images since it provides faster processing when compared to others image matching such as template matching technique [25]. 


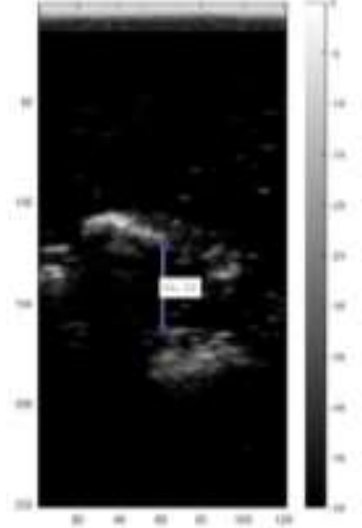

(a)

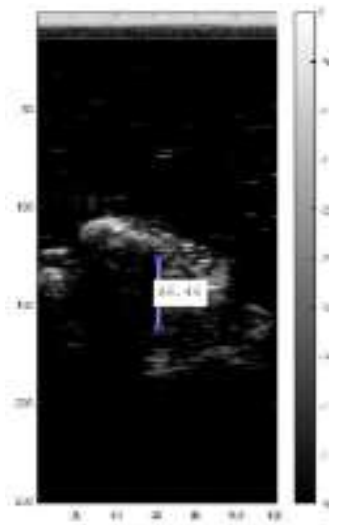

(d)

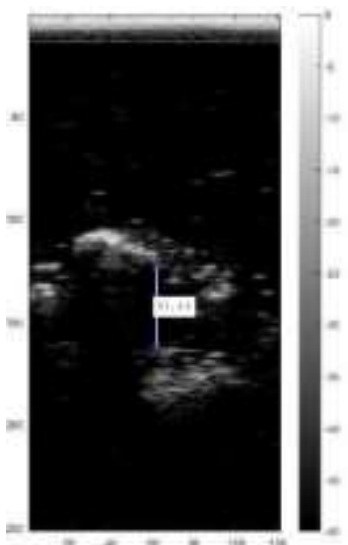

(b)

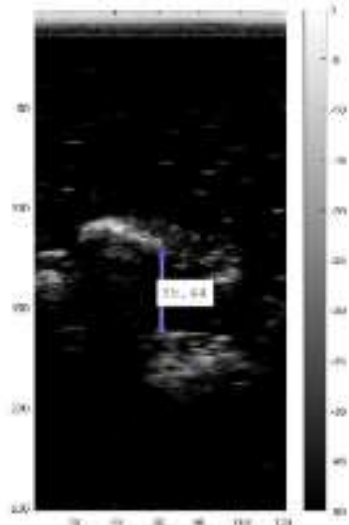

(e)

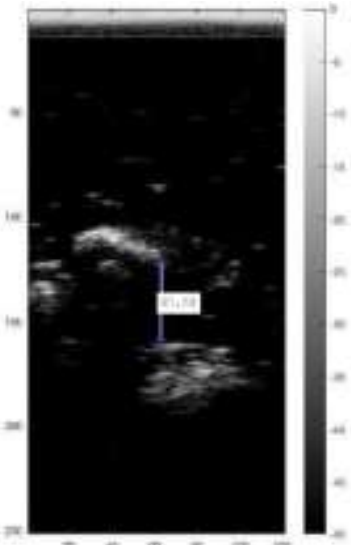

(c)

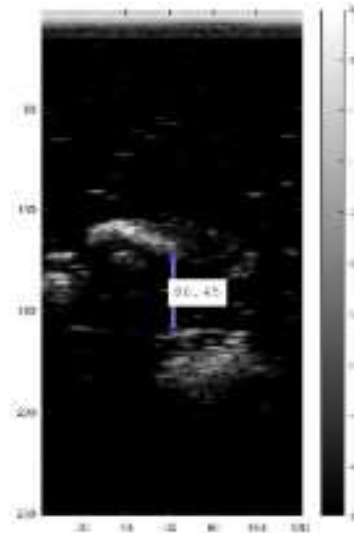

(f)

Figure 8. Distance valve measuring in pixel value; (a) Frame 1; (b) Frame 7; (c) Frame 15; d) Frame 25; (e) Frame 35; (f) Frame 51

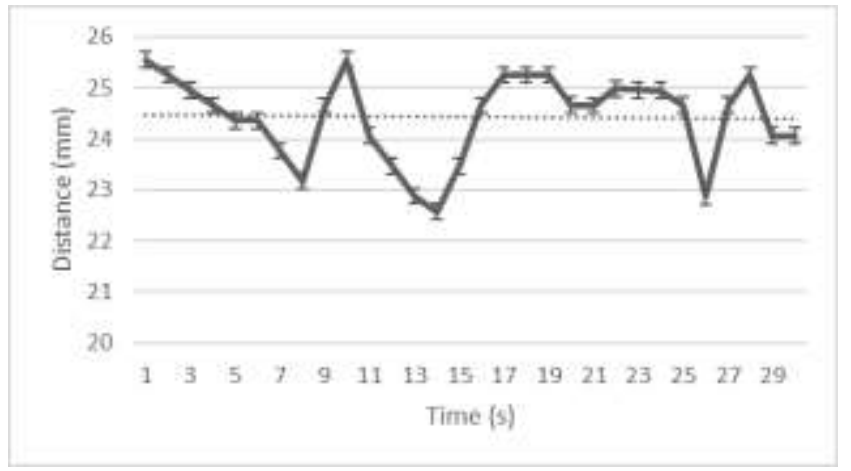

Figure 9. Changes of phantom valve distance along the time (s)

Different techniques were proposed in literature that consists of improveable parameters. Most of the discussed techniques applied ultrasound Doppler concept to measure the blood velocity. Gaining a new modern technique, deep-learning model is also designed for image classification to detect the early stage of disease in medical images [26]. Several reports have also explained the tracking techniques used for other application than the heart diagnostics. However, from some other views, tracking technique is negotiable to replace with segmentation practices in medical imaging [27]. Computer-aided segmentation becomes popular to be implemented as it has potential to avoid manual operation, [28] also reported the automated 
segmentation of scanned bone image that involved the evaluation of landmark points as the initial input to the assessment which similar to our proposed concept that proved the initial point is essential to be governed. Here, the determination of the points dependently to capture the bone shape is somehow being conducted manually. In this paper of the heart valve recognition, we have utilized the eigenvalue calculation that lead the selection of the points which increase the merit of the implemented technique

\section{CONCLUSION}

Cardiac diseases are a major health concern worldwide. In particular, useful information on the cardiac function can be extracted by analyzing of echocardiography sequences. However, echocardiographic images are difficult to analyze and depends mostly on the operator's skills and lifetime experiences to obtain a clear and better image. Unclear images may result in false diagnosis due to the tendency of existing speckle noise. In this study, a validation of work on proposed valve tracking technique is discussed. The results yielded the performance of proposed tracking technique by obtained $89 \%$ to $100 \%$ range of valve distance between the true measured and constructed valve distance phantom. This finding shows the proposed method reported in has good intention to predict the abnormality of blood flow in left side of heart. This method is proposed generally to dealing with the problem of highly cost in traditional technique of image registration. KLT utilizes information on spatial intensity to control looking for the position yielding the most exact match. It is a lot faster than traditional technique for checking lesser plausible matches between the images. However, based on the experiment, the accuracy of the technique decreases over the time. As a recommendation, segmentation on the mitral valve is advised in order to keep and maintain the information of location and structure. Moreover, this work has high possible for further improvement by comparing the accuracy between original images and preprocessed image. The point in distance measuring between valves should also be added.

\section{ACKNOWLEDGEMENTS}

This work is supported by Ministry of Higher Education Malaysia under Fundamental Research Grant Scheme Vot No. FRGS/1/2018/TK04/UTHM/02/24. The authors would like to express their gratefulness for the support and generosity from Universiti Tun Hussein Onn Malaysia (UTHM), Pantai Hospital Klang, and University of Toyama for providing the space doing the experiment and analysis to complete this work.

\section{REFERENCES}

[1] W. A. Hassan and H. H. Ebaid, "Assessment of Left Ventricular Function and Aortic Elastic Properties in Patients with Bechet's Disease Using Conventional and Tissue Doppler Echocardiography," The Egyptian Rheumatologist, vol. 38, no. 1, pp. 47-52, 2016, doi: 10.1016/j.ejr.2015.04.005.

[2] J. Spühler, J. Hoffman, U. Gustafsson, M. G. Larson, and P. Vesterlund, "Simulation of Blood Flow in the Left Ventricle Using Moving Geometries Based on Ultrasound Measurements," Elsevier, vol. 1, pp. 232-237, 2012.

[3] Z. Tang, Y. Fan, Y. Wang, Ch. Jin, K. Kwok, and A. P. Lee, "Mitral Annular and Left Ventricular Dynamics in Atrial Functional Mitral Regurgitation: A Three Dimensional and Speckle-Tracking Echocardiography Study," Journal of the American Society of Echocardiography. vol. 32, no. 4, pp. 503-513, 2019, doi: 10.1016/j.echo.2018.11.009.

[4] P. M. Shah, "Current Concepts in Mitral Valve Prolapse--Diagnosis and Management," Journal of Cardiology, vol. 56, no. 2, pp. 125-133, 2010, doi: 10.1016/j.jjcc.2010.06.004.

[5] J. M. Gardin, "Pulsed Doppler Ehocardiography: An Historical Perspective," Journal of the American Society of Echocardiography, vol. 31, no. 12, pp. 1330-1343, 2018, doi: 10.1016/j.echo.2018.09.013.

[6] R. Nagaoka, K. Ishikawa, M. Mozumi, M. Cinthio, and H. Hasegawa, "Basic Study on Estimation Method of Wall Shear Stress in Common Carotid Artery using Blood Flow Imaging," Japanese Journal of Applied Physics, vol. 59, 2020, doi: 10.35848/1347-4065/ab87f2.

[7] M. Mozumi, R. Nagaoka, and H. Hasegawa, "Utilization of Singular Value Decomposition in High-Frame Rate Cardiac Blood Flow Imaging," Japanese Journal of Applied Physics, vol. 58, 2019, doi: 10.7567/13474065/ab1131.

[8] H. Hasegawa, "Analysis of Arterial Wall Motion for Measurement of Regional Pulse Wave Velocity," Japanese Journal of Applied Physics, vol. 57, 2018, doi: 10.7567/JJAP.57.07LF01.

[9] S. Moral, E. Ballesteros, M. Huguet, A. Panaro, J. Palet, and A. Evangelista, "Differential Diagnosis and Clinical Implications of Remnants of the Right Valve of the Sinus Venosus." Journal of the American Society of Echocardiography, vol. 29, no. 3, pp. 183-194, 2016, doi: 10.1016/j.echo.2015.11.018.

[10] N. L. E. Okiwelu, F. Ramponi, S. Thakur, T. Vo, and J. Edwards, "Tissue Valve-over-valve Implantation in Previous Mechanical Bentall," Journal of Cardiac Surgery, vol. 35, no. 12, pp. 3545-3647, 2020, doi: 10.1111/jocs.14911. 
[11] G. L. Yost, T. J. Royston, G. Bhat, and A. J. Tatooles, "Acoustic Characterization of Axial Flow Left Ventricular Assist Device Operation in Vitro and in Vivo," ASAIO Journal, vol. 62, no. 1, pp. 46-55, 2016, doi: 10.1097/MAT.0000000000000307.

[12] T. L. Liao, H. C. Chen, and J. J. Yan, "Design of Real Time Face Position Tracking and Gesture Recognition System Based on Image Segmentation Algorithm," Journal of Network Intelligence, vol. 5, no. 4, pp. 226-239, 2020.

[13] J. J. Ali, N. M. Shati, and M. T. Gaata., "Abnormal Activity Detection in Surveillance Video Scenes," TELKOMNIKA Telecommunication, Computing, Electronics and Control, vol. 18, no. 5, pp. 2447-2453, 2020, doi: 10.12928/TELKOMNIKA.v18i5.16634.

[14] S. Vujasinovi'c, S. Becker, T. Breuer, S. Bullinger, N. Scherer-Negenborn, and M. Arens, "Integration of the 3D Environment for UAV Onboard Visual Object Tracking," Applied Sciences, vol. 10, no. 21, pp 7622, 2020, doi: 10.3390/app10217622.

[15] P. Mountney, D. Stoyanov, and G. Yang, "Three-Dimensional Tissue Deformation Recovery and Tracking," IEEE Signal Processing Magazine, vol. 27, no. 4, pp. 14-24, July 2010, doi: 10.1109/MSP.2010.936728.

[16] A. Ihsan, N. Fadillah, and C. Gunawan, "Acehnese Traditional Clothing Recognition based on Augmented Reality Using Hybrid Tracking Method," Indonesian Journal of Electrical Engineering and Computer Science", vol. 20, no. 2, pp. 1030-1036, 2020, doi: 10.11591/ijeecs.v20.i2.pp1030-1036.

[17] L. Mihaylova, P. Brasnett, N. Canagarajah, and D. Bull, "Object Tracking by Particle Filtering Techniques in Video Sequences," Advances and Challenges in Multisensor Data and Information Processing, vol. 8, pp 260-168, 2007.

[18] H. Kato and M. Billinghurst, "Marker tracking and HMD calibration for a video-based augmented reality conferencing system," Proceedings 2nd IEEE and ACM International Workshop on Augmented Reality (IWAR'99), 1999, pp. 85-94, doi: 10.1109/IWAR.1999.803809.

[19] N. H. Barnouti, M. H. N. Al-Mayyahi, and S. S. M. Al-Dabbagh, "Real-Time Face Tracking and Recognition System Using Kanade-Lucas-Tomasi and Two-Dimensional Principal Component Analysis," 2018 International Conference on Advanced Science and Engineering (ICOASE), 2018, pp. 24-29, doi: 10.1109/ICOASE.2018.8548818.

[20] C. Tomasi and T. Kanade. "Detection and Tracking of Point Features," Technical Report CMU-CS-91-132, Carnegie, Mellon University, 1991.

[21] L. F. Mahadi, N. Ibrahim, M. T. Zaluwi, and M. H. S. M. Johan, "Performance Evaluation on Mitral Valve Motion Feature Tracking Using Kanade-Lucas-Tomasi (KLT) Algorithm-Based Eigenvalue Measurement," AIP Coference Proceeding, vol. 2173, no. 1, 2019, doi: 10.1063/1.5133917.

[22] L. F. Mahadi, N. Ibrahim, M. T. Zaluwi, and M. H. S. M. Johan, "Feature Detection of Mitral Valve Based on Image Processing Technique: A Review," Journal of Physics: Conference Series, vol. 1372, pp. 1-6, 2019, doi: 10.1088/1742-6596/1372/1/012071.

[23] I. A. Fiqhi, R. Diana, and I. Wita, "Hijaiyah Letters Sign Language Recognition for Deaf and Hearing Impaired Chidren Based on Principle Component Analysis Method," Journal of Physics, vol. 1569, pp. 1-9, 2020, doi: 10.1088/1742-6596/1569/3/032077.

[24] V. Jalaja and G. S. G. N. Anjaneyulu, "Face Recognition by using Eigen Face Method," International Journal of Scientific and Technology Research, vol. 9, no. 3, pp. 961-966, 2020.

[25] Y. Honjo, H. Hasegawa, and H. Kanai, "Accurate ultrasonic measurement of myocardial regional strain rate at high temporal and spatial resolutions," 2008 IEEE Ultrasonics Symposium, 2008, pp. 1995-1998, doi: 10.1109/ULTSYM.2008.0492.

[26] M. S. Croock, S. D. Khuder, A. E. Korial, and S. S. Mahmood, "Early Detection of Breast Cancer using Mammography Images and Software Engineering Process," TELKOMNIKA Telecommunication, Computing, Electronics and Control, vol. 18, pp. 1784-1794, 2020, doi: 10.12928/TELKOMNIKA.v18i4.14718.

[27] N. Sharma and L. M. Aggarwal, "Automated Medical Image Segmentation Techniques," Journal of Medical Physics, vol. 35, no. 1, pp. 3-14, 2010, doi: 10.4103/0971-6203.58777.

[28] E. Rachmawati, Jondri, K. N. Ramadhani, A. H. S. Kartamihardja, A. Achmad, and R. Shintawati, "Automatic Whole-Body Bone Scan Image Segmentation Based on Constrained Local Model," Bulletin of Electrical Engineering and Informatics, vol. 9, no. 6, pp. 2526-2537, 2020, doi: 10.11591/eei.v9i6.2631.

\section{BIOGRAPHIES OF AUTHORS}

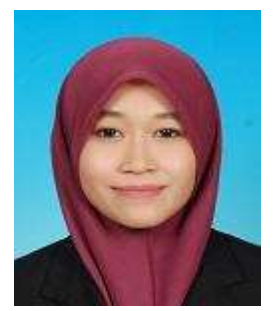

Lina Farhana Mahadi was born in Muar, Johor, Malaysia in 1992. She graduated Bachelor Degree from University Tun Hussein Onn Malaysia in Electrical and Electronic Engineering in 2015. She received M.E degree from University Tun Hussein Onn Malaysia in Electrical Engineering in 2017. She is currently a $\mathrm{PhD}$ candidate from University Tun Hussein Onn Malaysia in Electrical Engineering. Her main research interest is on medical electronic field and her current project mainly on image processing and image tracking. Lina is member of IEEE, IEEE Women in Engineering, and IEEE SIGHT. 

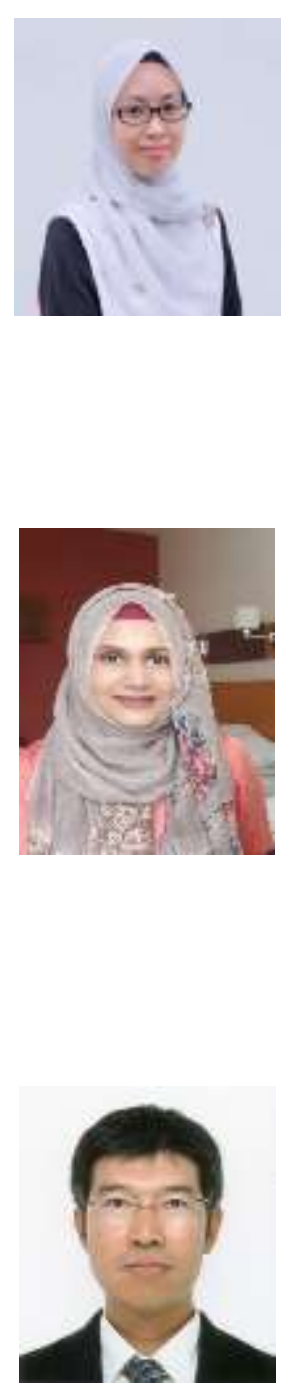

Nabilah Ibrahim is an associate professor of electrical and electronic engineering at University Tun Hussein Onn Malaysia (UTHM). She received the B. Eng in Telecommunication Engineering and M. Eng in Computer Sciences both from Shibaura Institute of Technology Japan. She holds a doctorate degree in Electronic Engineering which she received from Tohoku University Japan. During her work in UTHM, she served as Head of Department of International Office from 2015-2017 and currently she served as Editor-in-Chief of International Journal of Integrated Engineering (IJIE). Dr. Nabilah involves in Biomedical Electronic research area which include image processing, digital signal processing, and sensor and IOT application medical. She also involves in IEEE Malaysia Section as Honorary Secretary and serves as Executive Committee of IEEE EMBS Chapter. She is a member of IET and BEM.

Shahnoor Shanta received the B.Sc. (Hons.) and M.Sc. degrees in Applied Physics and Electronics from the University of Dhaka, Bangladesh. She also received the MSc in Communications and Signal Processing from Imperial College London and the Ph.D. degree in Statistical Signal Processing in Classification and Pattern Recognition from the Department of Automatic Control and System Engineering, University of Sheffield, U.K. She is an Associate Lecturer in Computer Science and Applied Computing at the School of Computing and Digital Media, London Metropolitan University (LMU) since 2020. Prior to joining the LMU, she worked as Guest Lecturer for the MEng in Connected and Autonomous Vehicle program, at the Institute of Technology Sligo, Ireland in 2020-2021. During 2015-2018, she worked as a Senior Lecturer in Electronics in University Tun Hussein On Malaysia, which followed her postdoctoral research at the School of Electrical Engineering and Computer Science, University of Ottawa, Canada during 2012-2015. Dr. Shanta joined as a Lecturer in Applied Physics and Electronics at the University of Dhaka following her MSc from the same Institution and worked there for several years up until her postdoctoral career. Some of her research interests include statistical signal processing and pattern recognition, speech and audio signal processing, multi-modal sensor fusion, brain computer interface, biomedical signal, and image processing. Furthermore, her research interests expand to Integration of IoT and Cloud Computing.

Hideyuki Hasegawa (IEEE Member) received the B.E. degree from Tohoku University, Sendai, Japan, in 1996. He received the Ph.D. degree from Tohoku University in 2001. He received the research fellowships for young scientists (DC1) and (PD) from the Japan Society for the Promotion of Science in 1998 and 2001, respectively. He became a research associate in 2002, lecturer in 2005, and associate professor in 2007 at the Graduate School of Engineering, Tohoku University. From 2008, he became an associate professor at the Graduate School of Biomedical Engineering, Tohoku University. He has been a professor at the Graduate School of Science and Engineering for Research, University of Toyama from 2015. His research interest is medical ultrasonics. He serves as a member of the technical program committee of the IEEE International Ultrasonics Symposium and an associate editor for IEEE Transactions on Ultrasonics, Ferroelectrics, and Frequency Control. 